\title{
Application of the coupled escape probability method to spherical clouds
}

\author{
Y. J. Yun, Y.-S. Park, and S. H. Lee \\ Department of Physics and Astronomy, Seoul National University, Korea \\ e-mail: yyzoo@astro.snu.ac.kr \\ Received 30 June 2009 / Accepted 21 August 2009

\begin{abstract}
Aims. We present applications of one of the novel radiative transfer methods, called the coupled escape probability method, to spherical clouds. It provides efficient tools for analyzing molecular lines, but its implementation is limited to plane-parallel systems. For more general uses, we extend its capability to spherical systems.

Methods. The spherical geometry means that the derivation of mean intensity has to be conducted numerically, whereas it was done more analytically in the original work. The new method was applied to spherical clouds and cores with various conditions, and systems also investigated.

Results. While this method is more complicated than the original method, it maintains the underlying simplicity and practicality. The excitation conditions derived for the various conditions are compared with those of the Monte Carlo methods, and the results are found to be in good agreement with each other. The new method is more efficient in computing time and number of iterations.
\end{abstract} \\ with moderate systematic motion are tested in addition to static systems. The case of molecular transitions with hyperfine overlap is
}

Key words. line: formation - radiative transfer - methods: numerical - ISM: molecules

\section{Introduction}

The progress of receiver technologies and the exploitation of high and dry sites have enabled radio observations at higher frequencies. Molecular transitions at such frequencies involve, in general, energy levels that are highly excited, thus reflecting the physical conditions of hot and/or dense regions in clouds or cores. Since populations will be distributed over a wide range of energy levels in this case, simply relying on the analysis of one or two transitions and/or on unrealistic assumptions will result in serious errors when deriving physical quantities. Thus, multitransitional studies of molecular lines are crucial. This is the area where the non-LTE radiative transfer and excitation (RT hereafter) models can play an important role in calculating physical quantities such as column density and molecular abundances (van der Tak et al. 2007). The models also help for inferring velocity fields inside molecular clouds/cores through analysis of the line shape (e.g., Zhou et al. 1993; Lee et al. 2007).

Among these is the large velocity gradient (LVG) model, which has long been used for its simplicity and robustness (Goldreich \& Kwan 1974; van der Tak et al. 2007). It actually solves the RT problem locally, based on the assumption of the large systematic motion. However, because this type of motion is very rare, the RT problem cannot be solved locally. Moreover, the model does not make use of information contained in the line shape. The first rigorous non-LTE treatment of molecular lines was made by Leung \& Liszt (1976), who solved the non-LTE RT model using the quasi-diffusion method. This model can provide both line profiles and excitation conditions, but cannot treat velocity fields properly except for microturbulence. Although there have been several RT models (van Zadelhoff et al. 2002, and references therein), the Monte Carlo method introduced by Bernes (1979) may be the most popular one, even to this day. The model traces the fate of real photons that are replaced by a much smaller number of model photons that are spontaneously generated inside and come from outside. Then, the mean radiation field and, accordingly, a number of (de-)excitations are readily calculated and are fed into the statistical equilibrium equation. The whole process is repeated via the $\Lambda$-iteration until convergence is obtained. Although noise is inherent in this model, the velocity field can be taken into account properly, and extension to a higher dimension is conceptually easy. This method has been expanded into three-dimensional ones (Park \& Hong 1995; Juvela 1997), improved to achieve better convergence with the accelerated $\Lambda$-iteration (Hogerheijde \& van der Tak 2000), or both (Wada \& Tomisaka 2005).

An interesting model of radiative transfer has been presented by Elitzur \& Asensio Ramos (2006). By adopting the zone-based gridding in a plane-parallel system, they derive the equations of statistical equilibrium, which are fully consistent with the radiative transfer based on the assumption that all physical properties are constant within each zone. With this assumption, the equation of radiative transfer is solved explicitly and analytically, and can then be easily merged into the level population equations. These equations are linearized and globally solved. This method is called coupled escape probability (CEP) because the different zones are coupled through the net radiative bracket (Athay \& Skumanich 1971), which resembles the standard escape probability expression.

A method based on the Gauss-Seidel algorithm presented by Trujillo Bueno \& Fabiani Bendicho (1995) and extended by Asensio Ramos \& Trujillo Bueno (2006) is also noteworthy. The basic concept of the algorithm is to update level populations and mean intensity, $\bar{J}$, whenever possible, in the course of sweeping 
grids from center to outer boundary. Daniel \& Cernicharo (2008) introduce several assumptions into the original Gauss-Seidel algorithm to reduce the computational time and applied the algorithm to the problem of $\mathrm{N}_{2} \mathrm{H}^{+}$hyperfine lines in a spherical system.

We have tried to apply the CEP method to the spherical system. In contrast to the plane-parallel system, it will be difficult to make use of the analytical approach of the CEP method in the spherical system. More numerical works are necessary, but the CEP concept will be weakened. However, the RT equation is still readily and explicitly solved since each zone is constant in physical quantities. Consequently, the system could be linearized and solved efficiently with, for example, the Newton method. The CEP spherical model is applied to clouds both with and without a velocity field for the $\mathrm{CO}$ molecule. In addition, the excitation of molecules with hyperfine splitting, such as $\mathrm{HCN}$, is investigated and compared with those from the Monte Carlo method. We describe the mathematical formulations in Sect. 2 and present results in Sect. 3. The discussion and conclusions are given in Sects. 4 and 5, respectively. Our final goal is to provide a handy tool for analyzing multi-transitional molecular lines with high fidelity and efficiency for spherical geometry.

\section{Mathematical formulation}

\subsection{Radiative transfer}

Here, we describe the mathematical formulation of radiative transfer in a spherical molecular cloud/core. For numerical work, the sphere is divided into many concentric shells, as usual. We assume that physical conditions are uniform within each zone. This assumption is not new and has been adopted in the Monte Carlo method (Bernes 1979), but it plays a crucial role in the CEP method. This assumption may be relaxed into a gridbased one, and a parabolic variation may be used for better realization of systems by sacrificing computation time and simplicity (Daniel \& Cernicharo 2008).

Thus, specific intensity $I\left(\tau_{v}\right)$ is readily calculated at a position in a zone along an arbitrary direction,

$I_{v}\left(\tau_{v}\right)=I_{\mathrm{b}} \mathrm{e}^{-\tau_{v}}+S\left(1-\mathrm{e}^{-\tau_{v}}\right)$,

where $S$ is source function and $\tau_{v}$ is optical depth from the boundary of the zone to the position where $I_{v}$ is measured along the ray. The $I_{\mathrm{b}}$ is the value at the zone boundary. Since the intensity varies even within a zone along the ray path, it is necessary to find an average intensity that is representative of the zone and that will be used for the calculation of mean intensity (see Eq. (9)). We choose the following definition of the average intensity for a zone, $i$, and for a ray, $s$, following Elitzur \& Asensio Ramos (2006):

$$
\begin{aligned}
\bar{I}_{v}^{i} & =\frac{1}{\tau_{v}^{i}} \int_{0}^{\tau_{v}^{i}} I_{v}(t) \mathrm{d} t \\
& =I_{\mathrm{b}}^{i} \frac{1-\mathrm{e}^{-\tau_{v}^{i}}}{\tau_{v}^{i}}+S^{i}\left(1-\frac{1-\mathrm{e}^{-\tau_{v}^{i}}}{\tau_{v}^{i}}\right),
\end{aligned}
$$

where the $\tau_{v}^{i}$ is the total optical depth of the zone, $i$, along the ray. The $\tau_{v}^{i}$ is given by

$$
\begin{aligned}
\tau_{v}^{i} & =\tau^{i} \Phi^{i}(\Delta v) \\
& =\frac{1}{4 \pi} g_{u} B_{u l} E_{u l}\left(n_{l}^{i}-n_{u}^{i}\right) \ell^{i} \Phi^{i}(\Delta v),
\end{aligned}
$$

where $\ell^{i}$ is the path length along the ray, $n_{l}^{i}$ the level population per state in the $i$ th zone, $B_{u l}$ the Einstein coefficient for the transition between the level of $u$ and $l$, and $E_{u l}$ the energy separation between the levels. The absorption profile is

$\Phi^{i}(\Delta v)=\frac{\mathrm{e}^{-\left(\Delta v / \Delta v_{\mathrm{D}}^{i}\right)^{2}}}{\sqrt{\pi} \Delta v_{\mathrm{D}}^{i}}$,

where $\Delta v_{\mathrm{D}}^{i}$ is the Doppler width, i.e.,

$\frac{\Delta v_{\mathrm{D}}^{i}}{v_{0}}=\frac{\Delta v_{\mathrm{D}}^{i}}{c}=\frac{1}{c} \sqrt{\frac{2 k T^{i}}{m}+\xi^{2}}$.

In Eq. (5), $v_{0}$ is the frequency at line center, $k$ the Boltzmann constant, $T^{i}$ the kinetic temperature of the $i$ th zone, $m$ the molecular mass, and $\xi$ the microturbulence parameter. Also, $\xi$ may have zonal dependence. The absorption profile shown in Eq. (4) is normalized so that $\int \Phi^{i}(\Delta v) \mathrm{d} \Delta v=1$. The line source function of the $i$ th zone, between the level of $u$ and $l$ is

$S^{i}=\frac{A_{u l}}{B_{u l}} \frac{n_{u}^{i}}{n_{l}^{i}-n_{u}^{i}}$,

where $A_{u l}$ is the Einstein coefficient for the transition.

The boundary value, $I_{\mathrm{b}}^{i}$, depends on the ray direction, i.e., the sign of the angle cosine. The sign of the angle cosine changes when the ray passes through the $m$ th zone, which is the innermost zone passed by the ray (see Fig. 1). If the angle cosine at the outer boundary of the $i$ th zone is $-\mu$ (inwardly moving rays), where $\mu$ is always positive, then

$I_{\mathrm{b}}^{i}=I_{v,-\mu}^{i}=I_{\nu,-\mu}^{i+1} \mathrm{e}^{-\tau_{\nu}^{i+1}}+S^{i+1}\left(1-\mathrm{e}^{-\tau_{\nu}^{i+1}}\right)$.

The $I_{v, \pm \mu}^{i}$ is defined as the specific intensity at the outer boundary of the zone, $i$. The $I_{v,-\mu}^{i+1}$ can be derived by applying the same equation to the zone, $i+2$, recursively. By finally applying this equation to the boundary of the cloud, $I_{v,-\mu}^{i}$ is fully specified. In our work, we set the boundary value to the $2.7 \mathrm{~K}$ background radiation, i.e., $I_{v,-\mu}^{N}=B(2.7 \mathrm{~K})$. For $+\mu$ (outwardly moving rays),

$I_{\mathrm{b}}^{i}=I_{v,+\mu}^{i-1}=I_{v,+\mu}^{i-2} \mathrm{e}^{-\tau_{\nu}^{i-1}}+S^{i-1}\left(1-\mathrm{e}^{-\tau_{\nu}^{i-1}}\right)$.

This equation is applied successively down to the $m$ th zone, and after that the Eq. (7) should again be used. If, e.g., a (proto)star resides at the center, the inner boundary conditions are set by $I_{v,+\mu}^{1}$, and no further application of the Eq. (7) is necessary.

There may be various ways of numerically deriving the mean intensity of the $i$ th zone, averaged over both angles and frequencies to be used in the equation of statistical equilibrium. We adopt the following equation by making use of the averaged specific intensity of Eq. (2):

$\bar{J}^{i}=\int \frac{\mathrm{d} \mu}{2} \int \bar{I}_{\nu}^{i} \Phi^{i}(v) \mathrm{d} v$.

In the case of a finite slab, the double integration in Eq. (9) is performed and simply expressed in terms of escape probabilities (Elitzur \& Asensio Ramos 2006). However, in the spherical system, the analytical integration is not possible, so the integration must be performed numerically. As a result, the concept of escape probability is not introduced explicitly for the spherical systems. For the integration over a solid angle in Eq. (9), one calculates $\bar{I}_{v}^{i}$ for various incident angles. We set the incident angle cosine at the surface, $\mu^{N}=-\left(k / N_{\text {ray }}\right)^{1 / 2}, k=1, \ldots, N_{\text {ray }}$, where $N_{\text {ray }}$ is the number of rays. This distribution of $\mu^{N}$ makes more rays pass through the inner zones compared to the equal-spaced distribution of $\mu^{N}$. When a numerical integration method, such 
as the rectangular method, is used for the integration over a solid angle, weighting factors should be used because the distribution of $\mu$ is not equal-spaced in every zone. Larger numbers of rays will make the integration more accurate.

Another factor to consider is that the angle cosine, $\mu$, of a ray is not constant along a ray path, even in the same zone. Thus, as we have done for $\bar{I}_{v}^{i}$, we need to average the angle cosine to obtain a representative value, $\bar{\mu}^{i}$, for numerical integration. One way of deriving $\bar{\mu}^{i}$ is to average the angle cosine over the path length of the $i$ th zone as follows:

$\bar{\mu}^{i} \equiv \frac{1}{\ell^{i}} \int_{0}^{\ell^{i}} \mu \mathrm{d} s$.

After completing some algebra, it is found that $\bar{\mu}^{i}=\left(R^{i}-\right.$ $\left.R^{i-1}\right) / \ell^{i}$, where $R^{i}$ is the radial distance to the outer boundary of the $i$ th zone from the center. An alternative way of deriving $\bar{\mu}^{i}$ is to evaluate the angle cosine at the position where the specific intensity is equal to the mean specific intensity along the ray, i.e., $\mathrm{e}^{-\tau_{v}}=\left(1-\mathrm{e}^{-\tau_{v}^{i}}\right) / \tau_{v}^{i}$. This definition enables us to take into account that each infinitesimal section of the ray contributes to $\bar{I}_{v}^{i}$ by different amounts, depending on the optical depth. From this condition,

$\bar{\mu}^{i}=\frac{R^{i} \mu^{i}-\mathcal{L}^{i}}{\sqrt{\mathcal{L}^{i 2}+R^{i 2}-2 \mathcal{L}^{i} R^{i} \mu^{i}}}$,

where $\mathcal{L}^{i}=\bar{\ell}^{i}$ for the rays moving inwardly, $\mathcal{L}^{i}=\ell^{i}-\bar{\ell}^{i}$ for the rays moving outwardly, and

$\bar{\ell}^{i}=\frac{-\ln \left(\frac{1-\mathrm{e}^{-\tau_{v}^{i}}}{\tau_{v}^{i}}\right)}{\tau_{v}^{i}} \ell^{i}$.

The $\mathcal{L}^{i}$ is the path length along a ray from the outer boundary of the $i$ th zone to the position where the above condition is satisfied. The $\mu^{i}$ is the angle cosine between the ray and the radial vector at the outer boundary of the $i$ th zone (see Fig. 1). The mean angle cosine described in Eq. (11) can be different from transition to transition even in a zone for a ray. Moreover, $\bar{\mu}^{i}$ for a transition can vary during iteration. We adopt Eq. (11) instead of Eq. (10) in our calculations. While this choice obviously takes more CPU time, it appears more reasonable, as mentioned above. Usually, to get better numerical accuracy, there should be enough zones that are optically thin. In this case, the two definitions of $\bar{\mu}^{i}$ will lead to virtually the same result. We calculated the cloud models with the zones of $\tau_{v}^{i}<0.5$ using the two definitions of the mean angle cosine and found that the difference is indeed less than $1 \%$ in terms of the excitation temperature.

Finally, we are able to write the mean intensity integrated over absorption profile of the $i$ th zone as

$\bar{J}^{i}=\frac{1}{2 N_{\text {ray }} N_{f}} \sum_{s=1}^{N_{\text {ray }}} \sum_{f=1}^{N_{f}}\left[\bar{I}_{v,-\mu_{s}}^{i} W_{-\bar{\mu}_{s}}^{i}+\bar{I}_{v,+\mu_{s}}^{i} W_{+\bar{\mu}_{s}}^{i}\right] \Phi^{i}\left(\Delta v_{f}\right)$,

where $W_{-\bar{\mu}_{s}}^{i}$ and $W_{+\bar{\mu}_{s}}^{i}$ are the weighting factors for the $\bar{\mu}_{s}^{i}$ of the ray, $s$, moving inwardly and outwardly, respectively. In the frequency domain, $\Delta v_{f}$ is from $-5 \Delta v_{\mathrm{D}}$ to $5 \Delta v_{\mathrm{D}}$ with equal spacing. One may use quadrature summation for better accuracy with less summations, but should expect to encounter difficulties when velocity fields are taken into account. Normally, we carried out calculations with $N_{\text {ray }}=100$ and $N_{f}=30$. While it is possible to perform double summation by using random

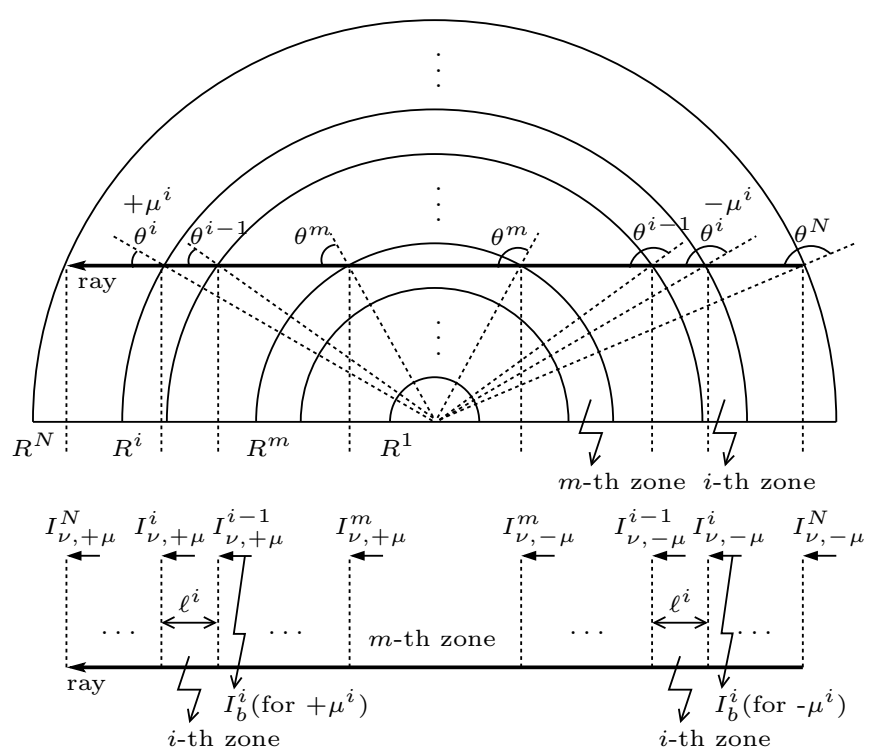

Fig. 1. Geometric quantities of the model.

numbers, as in Bernes (1979), we want to make the problem deterministic. If there are enough rays and the frequency spacing is narrow enough, the two approaches will be virtually identical (González-Alfonso \& Cernicharo 1993; Hogerheijde \& ven der Tak 2000).

\subsection{Statistical equilibrium}

One of the advantages of assuming that physical conditions are uniform in each zone is that one can explicitly see the dependencies of the mean intensity in a zone on level populations in other zones. This is possible since the mean specific intensity is analytically obtained, though it is within double summation. This enables us to linearize the rate equation and to solve it using the Newton method, after forming the Jacobian.

We denote the statistical weight of each level by $g_{l}$, and the population per sublevel in the zone, $i$ by $n_{l}^{i}$. The steady-state rate equations for a molecule with $L$ levels in the $i$ th zone are

$\frac{\mathrm{d} n_{k}^{i}}{\mathrm{~d} t}=\frac{1}{g_{k}}\left(-\sum_{l=1}^{L} R_{k l}^{i}+\sum_{u=1}^{L} R_{u k}^{i}\right)=0$,

where

$R_{u l}^{i}=A_{u l} n_{u}^{i} g_{u}+B_{u l} \bar{J}_{u l}^{i}\left(n_{u}^{i}-n_{l}^{i}\right) g_{u}+C_{u l}\left(n_{u}^{i}-n_{l}^{i} \mathrm{e}^{-E_{u l} / k T}\right) g_{u}$

These are $L-1$ independent equations for the $L$ unknown populations in each zone. We can make this system closed by using

$n^{i}=\sum_{k=1}^{L} g_{k} n_{k}^{i}$

which is the overall population in the zone, $i$ for the multi-level system.

Equations (14) and (16) form a set of nonlinear algebraic equations. For the system with $L$ levels and $N$ zones, the $M=$ $N L$ equations are given as

$f_{i}=0,(i=1,2, \ldots, M)$,

where, for $l \neq L$,

$f_{(i-1) L+l}=\frac{\mathrm{d} n_{l}^{i}}{\mathrm{~d} t},(l=1,2, \ldots, L-1)$, 
and for $l=L$,

$f_{i L}=n^{i}-\sum_{k=1}^{L} g_{k} n_{k}^{i}$

Usually, Eq. (17) is solved using the $\Lambda$-iteration method or its variants. However, the equation can be solved more efficiently using, e.g., the Newton method, because the dependencies of $R^{i}$ or specifically, $\vec{J}^{i}$, on the unknown variables are explicit in all terms of the equations. This method is written as $\boldsymbol{J} \cdot \delta \boldsymbol{n}=-\boldsymbol{f}_{0}$ using the Jacobian, $\boldsymbol{J}$, i.e.,

$$
\begin{gathered}
\sum_{i=1}^{M} \frac{\partial f_{1}}{\partial n_{i}} \delta n_{i}=-f_{0,1}, \\
\sum_{i=1}^{M} \frac{\partial f_{2}}{\partial n_{i}} \delta n_{i}=-f_{0,2}, \\
\vdots \\
\sum_{i=1}^{M} \frac{\partial f_{M}}{\partial n_{i}} \delta n_{i}=-f_{0, M} .
\end{gathered}
$$

We first assume level populations, then calculate the Jacobian, $\boldsymbol{J}$ and $\boldsymbol{f}_{0}$, and then make corrections, $\delta \boldsymbol{n}$, on the level populations. Subsequently, the Jacobian is evaluated again, and the whole process is repeated until convergence is achieved. Since the dependencies of $f_{i}$ on $n_{k}^{j}$ in other zones are explicitly known, iteration is faster than the $\Lambda$-iteration method. Actually, deriving the Jacobian for the first and third terms of the righthand side in Eq. (15) is straightforward, and the only concern is for the second term. This reduces to finding derivatives of the averaged specific intensity with respect to level populations,

$\frac{\partial \bar{I}_{v}^{i}}{\partial n_{k}^{j}}=\frac{\partial}{\partial n_{k}^{j}}\left[I_{\mathrm{b}}^{i} \frac{1-\mathrm{e}^{-\tau_{v}^{i}}}{\tau_{v}^{i}}+S^{i}\left(1-\frac{1-\mathrm{e}^{-\tau_{v}^{i}}}{\tau_{v}^{i}}\right)\right]$.

In light of Eqs. (7) and (8), both for $-\mu$ (moving inwardly) and for $+\mu$ (moving outwardly), Eq. (21) can be expressed as follows. For $-\mu$, there are three cases:

i) $j<i$;

$$
\frac{\partial \bar{I}_{v}^{i}}{\partial n_{k}^{j}}=0
$$

ii) $j=i$;

$$
\frac{\partial \bar{I}_{v}^{i}}{\partial n_{k}^{j}}=\left(I_{v,-\mu}^{j}-S^{j}\right) \beta^{j^{\prime}}+S^{j^{\prime}}\left(1-\beta^{j}\right)
$$

iii) $j>i$;

$$
\frac{\partial \bar{I}_{v}^{i}}{\partial n_{k}^{j}}=\beta^{i} \prod_{t=i+1}^{j-1} E^{t}\left[\left(I_{v,-\mu}^{j}-S^{j}\right) E^{j^{\prime}}+S^{j^{\prime}}\left(1-E^{j}\right)\right] .
$$

For $+\mu$, there are also three cases:

i) $j<i$;

$$
\begin{aligned}
\frac{\partial \bar{I}_{v}^{i}}{\partial n_{k}^{j}}= & \beta^{i} \prod_{t=j+1}^{i-1} E^{t}\left[\left(I_{v,+\mu}^{j-1}-S^{j}\right) E^{j^{\prime}}+S^{j^{\prime}}\left(1-E^{j}\right)\right] \\
& +\beta^{i} \prod_{t=m}^{j-1} E^{t} \prod_{t=m+1}^{i-1} E^{t}\left[\left(I_{v,-\mu}^{j}-S^{j}\right) E^{j^{\prime}}+S^{j^{\prime}}\left(1-E^{j}\right)\right],
\end{aligned}
$$

ii) $j=i$;

$$
\begin{aligned}
\frac{\partial \bar{I}_{v}^{i}}{\partial n_{k}^{j}}= & \left(I_{v,+\mu}^{j-1}-S^{j}\right) \beta^{j^{\prime}}+S^{j^{\prime}}\left(1-\beta^{j}\right) \\
& +\beta^{j} \prod_{t=m}^{j-1} E^{t} \prod_{t=m+1}^{j-1} E^{t}\left[\left(I_{v,-\mu}^{j}-S^{j}\right) E^{j^{\prime}}+S^{j^{\prime}}\left(1-E^{j}\right)\right],
\end{aligned}
$$

iii) $j>i$;

$$
\frac{\partial \bar{I}_{v}^{i}}{\partial n_{k}^{j}}=\beta^{i} \prod_{t=m}^{j-1} E^{t} \prod_{t=m+1}^{i-1} E^{t}\left[\left(I_{v,-\mu}^{j}-S^{j}\right) E^{j^{\prime}}+S^{j^{\prime}}\left(1-E^{j}\right)\right],
$$

where $\beta^{i}=\left(1-\mathrm{e}^{-\tau_{\nu}^{i}}\right) / \tau_{v}^{i}$, and $E^{i}=\mathrm{e}^{-\tau_{\nu}^{i}}$. The derivatives on $S$, $\tau_{\nu}$, and $\beta$ are straightforward, so will not be iterated here.

As shown in Eqs. (22) through (27), the derivatives of the intensity vanish so only the derivatives of the optical depth and the source function are left. Then, derivatives of $\vec{J}^{i}$ are obtained from Eq. (13) by summing the derivatives on $\bar{I}^{i}$ with weighting factors.

The grand Jacobian matrix consists of $N \times N$ block matrices, and a block matrix is composed of $L \times L$ elements. Diagonal blocks represent radiative and collisional interactions among the energy levels in the same zones, while nondiagonal blocks involve nonlocal radiative interactions between different zones. The simpler the transition network, the sparser the Jacobian. The Jacobian is formed in this way and the nonlinear equations are solved using the Newton method.

\subsection{Overlap of hyperfine lines}

For the overlapping lines, the source function is no longer independent of frequency. Assuming that there are $h$ overlapping transitions, we denote the upper levels by $u_{1}, u_{2}, \ldots, u_{h}$ and the lower levels by $l_{1}, l_{2}, \ldots, l_{h}$ in the transitions. The source function of the $i$ th zone is given by

$$
S_{v}^{i}=\frac{\sum_{k=1}^{h} \tau_{u_{k} l_{k}}^{i} \Phi_{u_{k} l_{k}}^{i}(v) S_{u_{k} l_{k}}^{i}}{\sum_{k=1}^{h} \tau_{u_{k} l_{k}}^{i} \Phi_{u_{k} l_{k}}^{i}(v)}
$$

where $S_{u_{k} l_{k}}^{i}$ is the frequency-independent source function, which is the same as Eq. (6). The optical depth is also obtained using the summation of the line optical depths involved in the overlaps. They are given by

$\tau_{v}^{i}=\sum_{k=1}^{h} \tau_{u_{k} l_{k}}^{i} \Phi_{u_{k} l_{k}}^{i}(v)$

The intensity of the $i$ th zone, $\bar{I}_{v}^{i}$, is also obtained from Eq. (2), and the calculation of $\bar{J}^{i}$ for the overlapping lines uses the same process as for the lines without overlapping. The level populations can be calculated by solving the steady-state rate equations in the same way as they are calculated for the nonoverlapping lines. The Jacobian is also calculated analytically, and the calculation is simply lengthier because the source function and the optical depth have more terms compared to the case without the line overlaps. In summary, more levels are involved in the line intensity, so the Jacobian contains more nondiagonal elements compared to the nonoverlapping case. 


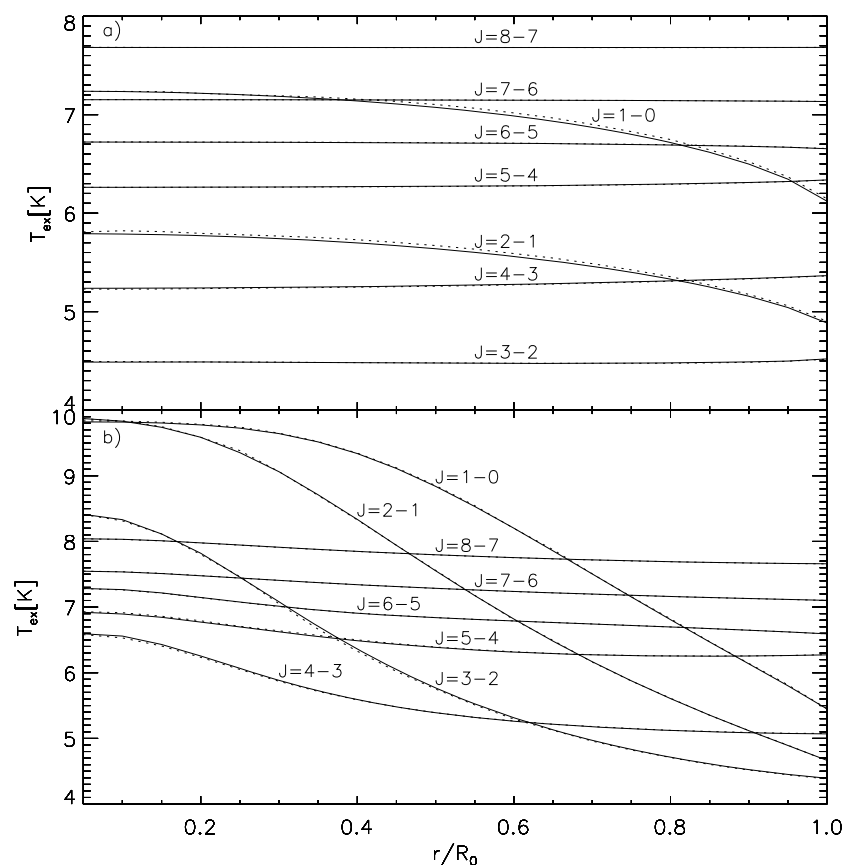

Fig. 2. Excitation temperatures of $\mathrm{CO}$ molecules against the normalized radial distance of static clouds with the kinetic temperature of $T_{\mathrm{k}}=$ $10 \mathrm{~K}$. Solid and dotted lines represent the results from our method and from the Monte Carlo method, respectively, a) represents the cloud of a constant density, $n_{\mathrm{H}_{2}}=10^{3} \mathrm{~cm}^{-3}$, and b) represents the cloud with a density gradient of $n_{\mathrm{H}_{2}}(r)=10^{4} /\left(1+\left(4 r / R_{0}\right)^{2}\right)$, where $R_{0}=10^{18} \mathrm{~cm}$.

\section{Results}

Our method is tested for a few cloud models in transitions both with and without line overlaps. Its results are compared with those from the Monte Carlo method.

\subsection{Static molecular clouds}

First, we test the method by applying it to the static clouds. The radius is $R_{0}=10^{18} \mathrm{~cm}$, the kinetic temperature is $T=10 \mathrm{~K}$, and the Doppler velocity width is set to $\Delta v_{\mathrm{D}}=1 \mathrm{~km} \mathrm{~s}^{-1}$. Two density models are used for this calculation: the first one has a constant density of $n_{\mathrm{H}_{2}}=10^{3} \mathrm{~cm}^{-3}$, and the second a density gradient of $n_{\mathrm{H}_{2}}(r)=10^{4} /\left(1+\left(4 r / R_{0}\right)^{2}\right) \mathrm{cm}^{-3}$. The relative abundance of $\mathrm{CO}$ to $\mathrm{H}_{2}$ is set to $10^{-5}$ for both cases. All models are calculated for $L=10$ lower levels of CO, and we use $N=20$ zones of equal spacing. Collisional coefficients by Flower (2001) and Wernli et al. (2006) are adopted for CO molecules. Figure 2 shows the results from our method and from the Monte Carlo method as well. We used the accelerated $\Lambda$-iteration method of Hogerheijde \& van der Tak (2000) for the Monte Carlo calculations. As expected, the excitation temperatures of lower transitions decrease toward the outer part of the cloud because of weak incident radiation. The excitation temperatures obtained from the two methods seem to be almost the same in all models. The difference amounts to $\lesssim 0.03 \mathrm{~K}$. The discrepancy may stem from differences in the integration of $\bar{I}_{v}$ to obtain the mean intensity, $\bar{J}$. The intrinsic noise of the Monte Carlo method is $0.02 \mathrm{~K}$ in this calculation. The line center optical depth of the thickest transition, $J=1-0$ is 3.5 for the first density model and 7.9 for the second density model. As we increase the optical depth by increasing the molecular hydrogen density, the agreement of the excitation temperature remains unaffected. Using our method, it took 7 iterations and about $35 \mathrm{~s}$ to achieve 0.1 percent relative

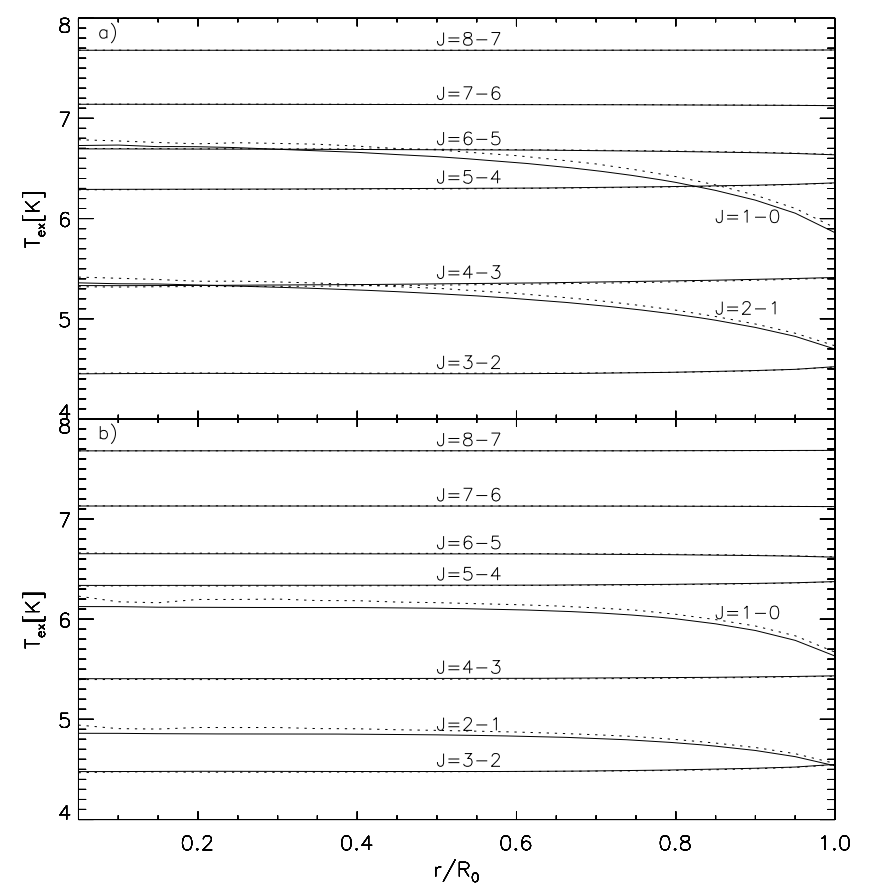

Fig. 3. Excitation temperatures of $\mathrm{CO}$ molecules against the normalized radial distance of a cloud under systematic motion with the kinetic temperature of $T_{\mathrm{k}}=10 \mathrm{~K}$. The density is constant with $n_{\mathrm{H}_{2}}=10^{3} \mathrm{~cm}^{-3}$. The linear velocity gradient exists from zero at the center to either a) $1.5 \Delta v_{\mathrm{D}}$ or b) $3 \Delta v_{\mathrm{D}}$ at the outer boundary. Results from our method (solid lines) and from the Monte Carlo method (dotted lines) are plotted.

accuracy, whereas 18 iterations and about 10200 s are necessary for the Monte Carlo method.

\subsection{Molecular clouds with moderate radial velocity gradient}

Next, we apply the method to molecular clouds of uniform density under systematic outward motion. The motion is linear with zero at the center and either $1.5 \Delta v_{\mathrm{D}}$ or $3 \Delta v_{\mathrm{D}}$ at the cloud boundary. The number of levels and the collisional coefficients are the same as above. We made the velocity gradient rather moderate so that the velocity in a zone is considered constant for the convenience of calculation. Figure 3 shows the distribution of the excitation temperatures for both methods. Optically thicker transitions seem to exhibit larger differences, but they still are less than $0.1 \mathrm{~K}$. However, differences in the way the velocity fields are dealt with may make the difference even greater. The velocity gradient makes the optical depth smaller, and the solutions are found for less iterations. Using our method, the iteration number and CPU time needed to reach a level of $10^{-3}$ relative accuracy are 6 and about $30 \mathrm{~s}$, respectively, whereas they are 18 and about 10200 s, respectively, for the Monte Carlo method. For larger velocity gradients, each zone should be divided further in order to take the velocity variation into account thoroughly, as Bernes (1979) did.

\subsection{Molecular clouds in the transitions of hyperfine HCN}

To validate the performance of the developed RT model further, we carried out calculations in the case of line overlap of HCN molecules. The radius is $R_{0}=10^{17} \mathrm{~cm}$, and the Doppler velocity and the total number of zones are the same as before. Density is constant with $n_{\mathrm{H}_{2}}=5 \times 10^{4} \mathrm{~cm}^{-3}$, and there is no velocity field in the cloud. The relative abundance of $\mathrm{HCN}$ to 


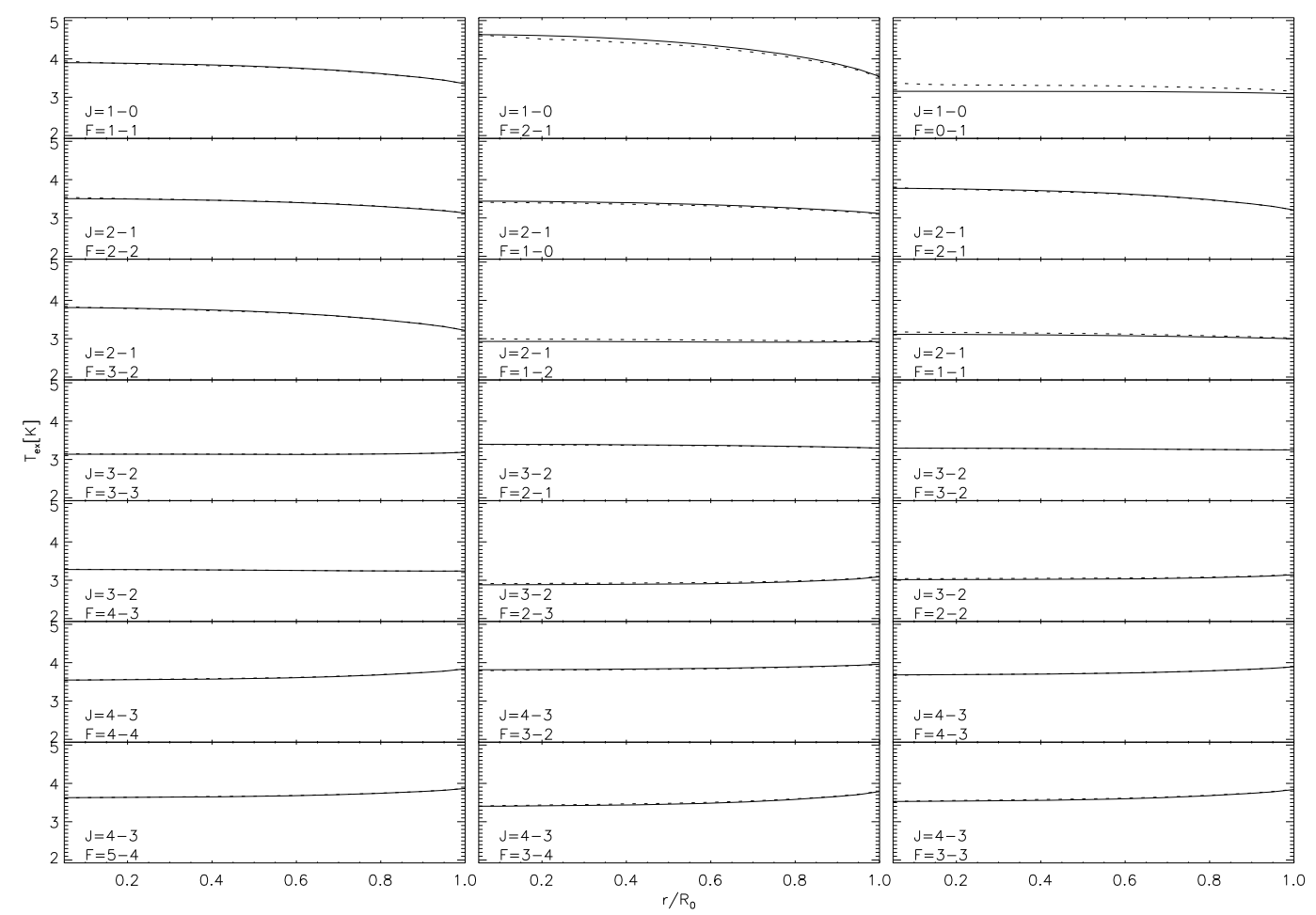

Fig. 4. Excitation temperatures of $\mathrm{HCN}$ molecules against the normalized radial distance of a static cloud with the kinetic temperature of $T_{\mathrm{k}}=10 \mathrm{~K}$. The radius of the cloud is $R_{0}=10^{17} \mathrm{~cm}$. Solid and dotted lines are the results from our method and from the Monte Carlo method, respectively.

molecular hydrogen is set to $10^{-8}$. We calculated the level populations for the lower 13 energy levels of HCN from $J=0$ to $J=4$, which have 21 hyperfine transitions. We used the same energy levels and Einstein A coefficients as in Park et al. (1999). Collisional coefficients were taken from Monteiro \& Stutzki (1986). The results were compared with those of the Monte Carlo method.

Figure 4 compares the excitation temperatures from this work and from the Monte Carlo method. Since the method used by Hogerheijde \& van der Tak (2000) does not deal with the case of hyperfine transition, we used the method developed by Park et al. (1999) instead. The largest difference amounts to $0.2 \mathrm{~K}$ for $J=1-0$ and $F=0-1$, and an overall agreement looks satisfactory. The maximum optical depth of the model at line center is 10.0 for $J=1-0$ and $F=2-1$. However, it seems that disagreements increase for higher optical depth. A similar situation is noted for the work by Daniel \& Cernicharo (2008). They conducted numerical computation with the Gauss-Seidel algorithm and compared the results with those of González-Alfonso \& Cernicharo (1993) and report that their results were qualitatively valid but deviated from those of González-Alfonso \& Cernicharo (1993) by 5-10\%. They suggest that the differences came from the choice of grid parameters, especially the number of radial grid points (see Daniel \& Cernicharo 2008). On the other hand, the gridding and molecular constants of our work are all the same as those of the Monte Carlo method. Any discrepancy seems intrinsic and further comparative studies are necessary.

\section{Discussion}

\subsection{Comparison with Monte Carlo method}

The method used to derive the mean intensity or the number of (de-)excitations, which is $B_{i j} \bar{J}$, is different for the two methods. In the Monte Carlo method, the total number of spontaneous photons is calculated and shot. As the photons travel, only absorptions are taken into account and the number of (de-)excitations counted. On the other hand, in the case of CEP, the mean specific intensity is derived rather conventionally in a deterministic manner, and the numerical integration is conducted to obtain the mean intensity.

The main difference lies in the method used to solve the statistical equilibrium equations. In the Monte Carlo method, (accelerated) $\Lambda$-iteration is utilized, while in CEP, it is the linearization method. Thanks to the uniformity condition of physical quantities in each zone, the mean intensity's dependence on the level population is known semi-analytically (Eq. (13)), and the Jacobian can be calculated accordingly. To summarize, the effect a level population in one zone has on the level population in another zone is known explicitly in CEP. The Jacobian enables a level population in one zone to feel the effect of infinitesimal variation of the level populations in the other zones (Elitzur \& Asensio Ramos 2006), which means final solutions can be found swiftly. For the optically thick lines, the Monte Carlo method has a slow convergence rate. Even for the optically very thin lines, the Monte Carlo method tends to generate too many new photons, which prevent fast processing (Hogerheijde \& van der Tak 2000). Because of the intrinsic noise in the Monte Carlo method, direct comparison of the performance is inadequate. However, it is clear that our RT model is handy and fast enough to accomplish one set of calculations in several iterations.

\subsection{Extension to multi-dimensions}

Our work is regarded as one of linearization methods. Actually, the linearization method that provides corrections for previous level populations in all zones and energy levels at a time is not new in stellar atmospheres (Mihalas 1978; Scharmer \& Carlsson 1985). It has also been adapted to the problem of spherical 
clouds (Rawlings \& Yates 2001). In our work, since the solution of radiative transfer equation is explicit, the linearization process as a whole is rather simple.

A main drawback of the global iteration is that it is usually limited to one-dimensional geometry, since the size of Jacobian becomes huge. It may be applicable to two-dimensional cases with special techniques, if the Jacobian matrix is sparse when the transition network is simple. However, it will practically be impossible to apply this method to three-dimensional geometry. It may be difficult even in the one-dimensional system for molecules involving many transitions like $\mathrm{CH}_{3} \mathrm{OH}$ and/or in high temperature. Methods like the accelerated $\Lambda$-iteration method that treat the statistical equilibrium equations grid by grid may be useful in this case (e.g., Daniel \& Cernicharo 2008).

\section{Conclusions}

We extended the applicability of the CEP method to spherical systems. The new method was tested for several cloud models with density or velocity gradients in simple $\mathrm{CO}$ transitions, and the case of hyperfine transition of $\mathrm{HCN}$ was investigated. The resulting excitation temperatures are found to be in good agreement with those of the Monte Carlo method. The concept of the escape probability in this method is vague in spherical geometry, and the spherical geometry introduces additional complexity in deriving the mean intensity integrated over frequency and its derivatives. However, the new method is practical and guarantees easy convergence down to $10^{-3}$, in terms of fractional level populations, in several iterations in most cases. The code is free to use and available at http://astro.snu.ac.kr/ yyzoo/ CEPsphere.
Acknowledgements. This work was supported by the Korea Research Foundation (KRF) grant funded by the Korea government (MEST) (No. 2009-0075189).

\section{References}

Asensio Ramos, A., \& Trujillo Bueno, J. 2006, EAS Publ. Ser., 18, 25 Athay, R. G., \& Skumanich, A. 1971, ApJ, 170, 605

Bernes, C. 1979, A\&A, 73, 67

Daniel, F., \& Cernicharo, J. 2008, A\&A, 488, 1237

Elitzur, M., \& Asensio Ramos, A. 2006, MNRAS, 365, 779

Flower, D. R. 2001, J. Phys. B, 34, 2731

Goldreich, P., \& Kwan, J. 1974, ApJ, 189, 441

González-Alfonso, E., \& Cernicharo, J. 1993, A\&A, 279, 506

Hogerheijde, M. R., \& van der Tak, F. F. S. 2000, A\&A, 362, 697

Juvela, M. 1997, A\&A, 322, 94

Lee, S. H., Park, Y.-S., Sohn, J., Lee, C. W., \& Lee, H. M. 2007, ApJ, 660, 1326 Leung, C. M., \& Liszt, H. S. 1976, ApJ, 208, 732

Mihalas, D. 1978, Stellar Atmospheres (San Francisco: W. H. Freeman and Company)

Monteiro, T. S., \& Stutzki, J. 1986, MNRAS, 221, 33

Park, Y.-S., \& Hong, S. S. 1995, A\&A, 300, 890

Park, Y.-S., Kim, J., \& Minh, Y. C. 1999, ApJ, 520, 223

Rawlings, J. M. C., \& Yates, J. A. 2001, MNRAS, 326, 1423

Scharmer, G. B., \& Carlsson, M. 1985, J. Comput. Phys., 59, 56

Trujillo Bueno, J., \& Fabiani Bendicho, P. 1995, ApJ, 455, 646

van der Tak, F. F. S., Black, J. H., Schöier, F. L., Jansen, D. J., \& van Dishoeck, E. F. 2007, A\&A, 468, 627

van Zadelhoff, G.-J., Dullemond, C. P., van der Tak, F. F. S., et al. 2002, A\&A, 395,373

Wada, K., \& Tomisaka, K. 2005, ApJ, 619, 93

Wernli, M., Valiron, P., Faure, A., et al. 2006, A\&A, 446, 367

Zhou, S., Evans II, N. J., Kömpe, C., \& Walmsley, C. M. 1993, ApJ, 404, 232 